\title{
Emission lines properties of the radio-loud quasar LAMOST J1131+3114 (Research Note)
}

\author{
Z. X. Shi ${ }^{1,2,3}$, G. Comte ${ }^{2,4}$, A. L. Luo ${ }^{2}$, Y. H. Zhao ${ }^{2}$, J. K. Zhao ${ }^{2,5}$, T. D. Oswalt ${ }^{5}$, and F. C. Wu ${ }^{1}$ \\ 1 National Laboratory of Pattern Recognition, Institute of Automation, Chinese Academy of Sciences, 100190 Beijing, PR China \\ ${ }^{2}$ Key Laboratory of Optical Astronomy, National Astronomical Observatories, Chinese Academy of Sciences, 100012 Beijing, \\ PR China \\ e-mail: lal@lamost.org, lal@nao.cas.cn \\ ${ }^{3}$ University of Chinese Academy of Sciences, 100049 Beijing, PR China \\ 4 Aix-Marseille Université, CNRS, Institut Pythéas, L.A.M., UMR 7326, 13388 Marseille Cedex, France \\ 5 Physics and Space Science Department, Florida Institute of Technology, Melbourne FL 32901, USA
}

Received 20 April 2013 / Accepted 24 February 2014

\begin{abstract}
The radio-loud quasar LAMOST J1131+3114 (Ton 580, B2 1128+315), showing asymmetrical profile in H $\beta$ and [O III] lines, has been selected from the LAMOST Pilot Survey for further study. We present an analysis of its emission lines by means of multiple Gaussian fitting, performed on LAMOST, Apache Point Observatory, and archival spectra. The broad line region, with an $\mathrm{H} \beta$ line of $F W H M \sim 4000 \mathrm{~km} \mathrm{~s}^{-1}$ has been fit with a double Gaussian, one of which is very broad and redshifted from the reference frame. The object is a likely Population B quasar. The [O III $\lambda \lambda 4959,5007$ lines profiles of the source have been formally fit with two Gaussian components, one of which is as broad as $\sim 700 \mathrm{~km} \mathrm{~s}^{-1}$. This broader component, if physically meaningful, appears to be subject to moderate velocity shock excitation. The forbidden line ratios correspond to a poorly populated area in standard diagnostic diagrams of AGN narrow line regions.
\end{abstract}

Key words. quasars: individual: LAMOST J1131+3114 - quasars: emission lines - galaxies: jets - galaxies: interactions galaxies: kinematics and dynamics

\section{Introduction}

The geometry and kinematics of the broad line region (BLR) in active galactic nuclei (AGNs) have been studied for a long time, but still remain unexplained. Zamfir et al. (2010) explore the properties of the $\mathrm{H} \beta$ emission line profile in a large, homogeneous, and bright sample of $N \sim 470$ low redshift quasars extracted from Sloan Digital Sky Survey (DR5). Their results give further support to the concept of two populations A and B (with line FWHM, respectively, narrower and broader than $4000 \mathrm{~km} \mathrm{~s}^{-1}$; Sulentic et al. 2007). Hu et al. (2008) report on a systematic investigation of the $\mathrm{H} \beta$ and Fe II emission lines in a sample of 568 quasars within $z<0.8$ selected from the SDSS and suggest the existence of an intermediate FWHM region.

Some sources have asymmetric and shifted $\mathrm{H} \beta$ profiles, suggesting that the BLR has a structure more complex than a single virialized component (Marziani et al. 1996, 2003). We have visually inspected all quasar spectra, which produced by the Pilot Sky Survey of LAMOST. An object exhibiting asymmetric [OIII] line profiles and an extended red wing of $\mathrm{H} \beta$ has been selected for further study: LAMOST J113109.5+311405.4 (hereafter LAMOST J1131+3114), a radio-loud quasar already known as Ton 580 or B2 $1128+315$.

We report on the analysis of several spectra of this source, including archival data. In Sect. 2, we briefly review the literature on this object, present our LAMOST and Apache Point Observatory (hereafter APO) spectra and summarize the archival data. In Sect. 3, we present the multicomponent spectral line fitting and its results. In Sect. 4, we discuss the characterization of BLR and narrow line region (NLR).

\section{Literature, new spectra and archival data}

\subsection{Summary of previous work}

The radio-loud quasar LAMOST J1131+3114, an UV excess source in the Tonantzintla survey (Ton 580: Iriarte \& Chavira 1957), was first detected at $408 \mathrm{MHz}$ in Colla et al. (1970) and listed in the Second Bologna Catalog (B2). Its redshift is $z=$ 0.289 (Hewitt \& Burbidge 1980). Classified as a single component radio source with a flat spectrum by Fanti et al. (1975), it has been mapped at $1.4 \mathrm{GHz}$ in the FIRST survey, where it exhibits a curved extended jet/lobe protruding out of the dominating compact radio core towards the east. Kimball et al. (2011) classify it as a J (core + jet) quasar and Snellen et al. (2002) as a flat spectrum radio quasar (FSRQ). Assuming isotropic emission, the radio luminosity at $1.4 \mathrm{GHz}$ is $\log L_{1400}=32.9$ for an optical ( $V$ band) luminosity of $\log L_{5500 \AA}=30.6\left(L\right.$ in $\left.\operatorname{erg~s}^{-1} \mathrm{~Hz}^{-1}\right)$. Unlike most FSRQ, optical variability and optical polarization remain undetected (see, e.g., Stalin et al. 2004a,b; Stockman et al. 1984) and the $1.4 \mathrm{GHz}$ polarized flux fraction is very low. From a 5 GHz VLBA map (Helmboldt et al. 2007), FIRST, and NVSS maps, a Fanaroff-Riley type II seems unlikely. The radio loudness (Kellermann et al. 1989) is $R \sim 80$ for the core alone and $R \geq 105$ for the whole source (Helmboldt et al. 2007). The spectral index is -0.63 between $74 \mathrm{MHz}$ and $8.4 \mathrm{GHz}$. This quasar should be a young, core dominated source using the classification of Zamfir et al. (2008).

Stockton (1978) measured redshifts of two faint galaxies at 7" SE and 35" SW from the quasar $(30 \mathrm{kpc}$ and $150 \mathrm{kpc}$ in projected distance) and found values very close to the quasar 
redshift, showing evidence for its belonging to a physical group of objects.

The optical spectrum of LAMOST J1131+3114 has been studied by several authors. Eracleous \& Halpern (1994) from a $6 \AA$ resolution spectrum covering $\mathrm{H} \alpha$ found a broad line with a full width at zero intensity of $22000 \mathrm{~km} \mathrm{~s}^{-1}$ and a FWHM of $4000 \mathrm{~km} \mathrm{~s}^{-1}$. Corbin (1997) shows a spectrum where what he calls the "blue bump" (yielding the UV excess in imagery) is obvious, with very weak Fe II broad lines. Marziani et al. (2003) studied the $\mathrm{H} \beta$ line range and confirmed a low value of $\mathrm{Fe}$ II blends for our object. They found an asymmetric $\mathrm{H} \beta$ line profile with a weak, redshifted barycenter, very broad component extending beyond $5000 \AA$ in rest frame. Grupe et al. (2004) also gave low values of $\mathrm{Fe}$ II equivalent width and $\mathrm{Fe} I \mathrm{I} / \mathrm{H} \beta$ ratios.

Finally, the X-ray properties of this source were studied using the Einstein Observatory (Shastri et al. 1993; and Wilkes et al. 1994), ROSAT (Brinkmann et al. 1995), XMM-Newton (Page et al. 2004), and Swift (Grupe et al. 2010). Page et al. (2004) showed evidence of a soft X-ray excess in the spectrum. Giommi et al. (2012) did not detect LAMOST J1131+3114 in $\gamma$-ray or submillimeter ranges using Fermi and Planck.

\subsection{New observations}

The LAMOST instrument is a multi-object spectroscopic facility able to take 4000 spectra of objects distributed across a field of 25 square degrees simultaneously in a single exposure (Cui et al. 2012). The spectral range extends from 3700 repeat unit to $9000 \AA$ with a resolution of $R=1800$. Prefiguring the large LAMOST survey of 10 million objects, a Pilot Survey was initiated on 2011 Oct. 24 for extensive performance evaluation. LAMOST J1131+3114, was observed on 2011 December 24, targeted by fiber 51 of spectrograph number 15 . The raw data have been reduced using the LAMOST data reduction pipeline and only a coarse relative flux calibration was applied (Luo et al. 2004, 2012).

Follow-up observations of LAMOST J1131+3114 were made with the Double Imaging Spectrograph on the ARC $3.5 \mathrm{~m}$ telescope at APO on 2012 June 15. The spectrograph was used in high-resolution mode ( $2 \AA /$ pixel) with a $1.5^{\prime \prime}$ slit $(6 \mathrm{kpc})$ oriented east-west. The gratings were centered at $6800 \AA$ (red) and $4400 \AA$ (blue), yielding wavelength coverages of 6200-7600 $\AA$ and 3800-5000 $\AA$, unfortunately slightly too red to cover completely the $\mathrm{H} \beta$ line profile of the source shotward of $4815 \AA$ (in rest frame).

The data were reduced with IRAF reduction procedures, following the usual path (bias, flats, HeNe arc, flux standard) with calibration data taken on the same night. The source HZ44 was used as the spectrophotometric flux standard star. Since the APO spectrum flux calibration is very consistent with the Marziani et al. spectrum, we extrapolated the missing region 4815-4700 ̊ from the latter.

\subsection{Archival data}

Except for the new LAMOST and APO data, we have used available archival spectra from Eracleous \& Halpern (1994), Marziani et al. (2003), and Grupe et al. (2004). The SDSS catalogue provided a spectrum (Plate 1974, MJD 53430, Fiber 174), but unfortunately data in the range $\mathrm{H} \beta$ - [O III] lines are missing. We note that the flux calibration of the Marziani et al. spectrum agrees closely with the SDSS spectrum for the flux densities of

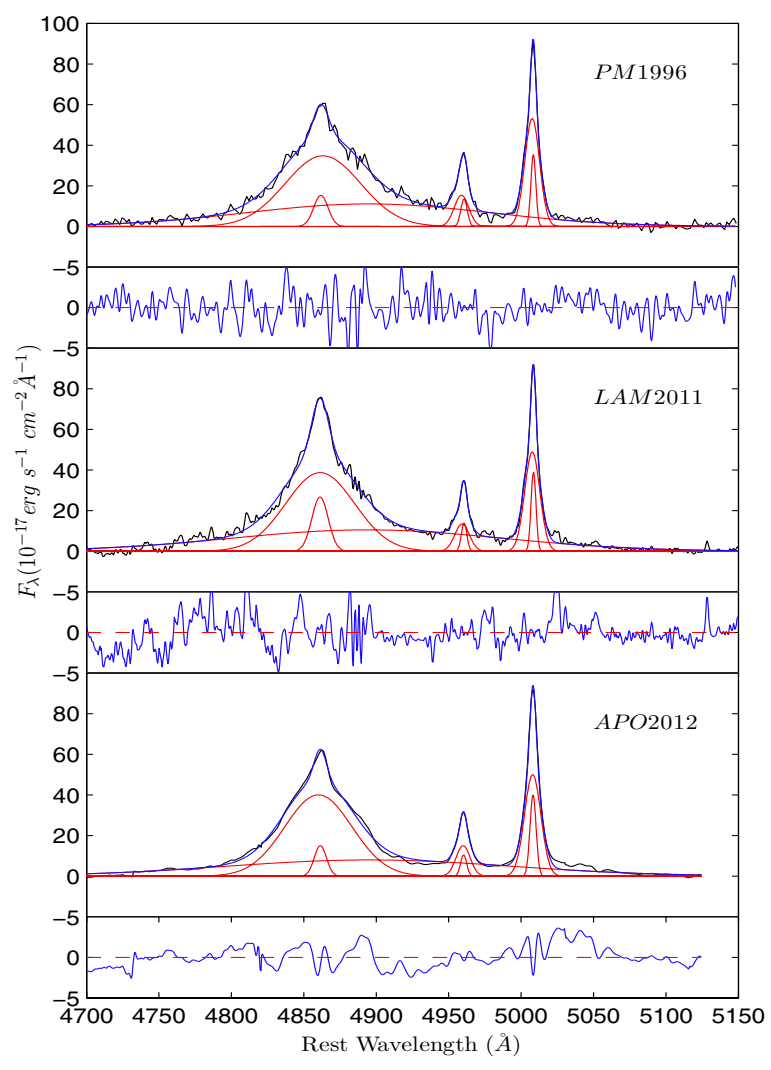

Fig. 1. $\mathrm{H} \beta$ and $[\mathrm{O} I \mathrm{II}]$ emission line fitting of LAMOST J1131+3114. PM1996: Marziani et al. (2003) (1996 May 22), LAM2011: LAMOST (2011 December 24), APO2012: Apache Point Obs. (2012 June 15). Black: original spectra, red: the Gaussian components, blue: sum of components. The bottom panels show the residuals.

$\mathrm{H} \gamma$ and [OIII] 24363 . After proper normalization on the peak flux density of [O III] $\lambda 5007$, the five spectra are very consistent, implying overall temporal stability in the emission lines fluxes across 15 years.

\section{Data analysis}

\subsection{Multicomponent fitting of $H \beta$ and [O III] lines}

The $\mathrm{H} \beta$ line of LAMOST J1131+3114 shows a sharp peak superimposed over a much broader and asymmetric profile, with a very broad and weak wing extending even beyond [O III] $\lambda 5007$. Owing to the Fe II emission weakness, we have assumed that the principal contributor to the local background below the [O III] lines is the $\mathrm{H} \beta$ red broad tail.

In Fig. 1, the profiles of both [O III] lines appear asymmetric with an extended wing on the blue side. A check of the PSF of LAMOST and APO spectrographs did not show any skewness in their PSFs, whose FWHM were locally measured at $3.96 \pm 0.02 \AA$ and $1.74 \pm 0.07 \AA$ at $\lambda=6400 \AA$. We chose the reference frame for the systemic redshift of the object as that defined by the central wavelength of the observed principal peak of the [O III] $\lambda 5007$ line. All spectra were first shifted back to this rest frame after conversion to the vacuum wavelength system when necessary. We measured $z=0.28946 \pm 0.00025$ (using a weighted average based on the spectral resolutions).

To model the $\mathrm{H} \beta$ profile, we built a three-Gaussian fit: a narrow component, a broad component $(\mathrm{BC})$, and a very broad component (VBC), the center of the third being redshifted with respect to the $\mathrm{BC}$ by around $2000 \mathrm{~km} \mathrm{~s}^{-1}$. The $\mathrm{BC}$ itself has 
Table 1. Average parameters of emission lines components derived from multi-Gaussian fit.

\begin{tabular}{|c|c|c|c|c|}
\hline \multirow[t]{2}{*}{ Comp. $^{a}$} & $\begin{array}{c}\text { Flux } \\
\left(10^{-17} \mathrm{erg} \mathrm{s}^{-1} \mathrm{~cm}^{-2}\right)\end{array}$ & $\begin{array}{c}V_{\text {offset }} \\
\left(\mathrm{km} \mathrm{s}^{-1}\right)\end{array}$ & $\begin{array}{l}F W H M \\
\left(\mathrm{~km} \mathrm{~s}^{-1}\right)\end{array}$ & \multirow[t]{2}{*}{ Src. ${ }^{b}$} \\
\hline & \multicolumn{3}{|c|}{$\mathrm{H} \beta$} & \\
\hline $\begin{array}{l}\text { BASE } \\
\text { BC } \\
\text { VBC }\end{array}$ & $\begin{array}{c}242 \pm 22 \\
2304 \pm 68 \\
2263 \pm 143\end{array}$ & $\begin{aligned}-80 & \pm 10 \\
-70 & \pm 20 \\
1960 & \pm 150\end{aligned}$ & $\begin{array}{c}720 \pm 40 \\
3540 \pm 60 \\
13350 \pm 320\end{array}$ & $\begin{array}{l}(1) \\
(1) \\
(1)\end{array}$ \\
\hline $\mathrm{VBC}$ & \multicolumn{3}{|c|}{ [O III] $\lambda 5007$} & \\
\hline \multirow[t]{2}{*}{ BASE } & $\begin{array}{l}176 \pm 26 \\
675 \pm 43 \\
\end{array}$ & $\begin{array}{c}10 \pm 10 \\
-30 \pm 10 \\
\end{array}$ & $\begin{array}{l}270 \pm 10 \\
750 \pm 20 \\
\end{array}$ & $\begin{array}{l}(2) \\
(2) \\
\end{array}$ \\
\hline & \multicolumn{3}{|c|}{ [O III] $\lambda 4959$} & \\
\hline \multirow[t]{2}{*}{$\begin{array}{c}\text { NC } \\
\text { BASE } \\
\end{array}$} & $\begin{array}{c}66 \pm 19 \\
182 \pm 31 \\
\end{array}$ & $\begin{array}{c}10 \pm 10 \\
-60 \pm 30 \\
\end{array}$ & $\begin{array}{l}300 \pm 60 \\
710 \pm 70 \\
\end{array}$ & $\begin{array}{l}(2) \\
(2) \\
\end{array}$ \\
\hline & \multicolumn{3}{|c|}{$\mathrm{H} \gamma$} & \\
\hline $\begin{array}{l}\text { BASE } \\
\text { BC } \\
\text { VBC }\end{array}$ & $\begin{array}{c}61 \pm 13 \\
638 \pm 44 \\
853 \pm 83\end{array}$ & $\begin{array}{c}40 \pm 40 \\
50 \pm 80 \\
630 \pm 180\end{array}$ & $\begin{array}{c}710 \pm 130 \\
3110 \pm 200 \\
9700 \pm 650\end{array}$ & $\begin{array}{l}\text { (3) } \\
\text { (3) } \\
\text { (3) }\end{array}$ \\
\hline VBC & \multicolumn{3}{|c|}{ [O III] $\lambda 4363$} & \\
\hline BASE & $115 \pm 16$ & $50 \pm 20$ & $720 \pm 80$ & (3) \\
\hline
\end{tabular}

Notes. ${ }^{(a)}$ NC: narrow component, BASE: a broader line base underlying $\mathrm{NC}$ in $[\mathrm{O} I \mathrm{II}], \mathrm{BC}$ : broad component, VBC: very broad component. (b) Weighted averages from spectra: (1) LAMOST, APO, Marziani et al. (2003), Grupe et al. (2004); (2): the same plus Eracleous \& Halpern (1994); (3) LAMOST and SDSS.

a redshift consistent with the adopted reference frame, which has a slight blueshift. The blue wing asymmetry of [O III] $\lambda \lambda 4959,5007$ lines prevented correct single-Gaussian modeling. We first tried two Gaussians of more or less similar FWHM, but better residuals were obtained using a significantly broader line base, as shown e.g., by Zamfir et al. (2010) (see their median spectrum of Type B1) underlying a narrower component (NC) and blueshifted (by about $50 \mathrm{~km} \mathrm{~s}^{-1}$ ) with respect to the latter. The final result of the simultaneous fit of the three lines $\mathrm{H} \beta$ and [O III] is given in Table 1 (where the errors are formal averages from several spectra) and is illustrated in Fig. 1 for the three best spectra.

\subsection{Other lines of LAMOST J1131+3114}

The very broad component of the $\mathrm{H} \beta$ emission is expected to be visible in all permitted lines of $\mathrm{H}$ and $\mathrm{He}$. In $\mathrm{H} \alpha$ it is clearly visible on the spectrum published by Eracleous \& Halpern (1994) and also on the SDSS spectrum, extending largely beyond the position of the $[\mathrm{S} I \mathrm{II}]$ lines. The $\mathrm{H} \alpha$ complex is expected to contain a narrow component, $\mathrm{BC}$ and $\mathrm{VBC}$ of $\mathrm{H} \alpha$, the two [NII] lines, and BC and VBC of the $\mathrm{He}_{\mathrm{I}}$ line at $6678 \AA$. We did not attempt to fit the spectrum with multiple Gaussian across this range because of numerical stability problems.

The $\mathrm{H} \gamma-$ [O III $\lambda 4363$ emission complex is only covered by LAMOST and SDSS spectra. We have made a tentative multicomponent fit of this complex, using 4 Gaussians, after a 5 Gaussians trial showed that the NC was not confidently detectable in [O III] line. The result is given in Table 1. From the residuals of the fit, we can derive upper limits of the total fluxes of a NC in $\mathrm{H} \gamma$ as $12.5 \times 10^{-17} \mathrm{erg} \mathrm{s}^{-1} \mathrm{~cm}^{-2}$ and in [O III] $\lambda 4363$ as $10 \times 10^{-17} \mathrm{erg} \mathrm{s}^{-1} \mathrm{~cm}^{-2}$.

From the SDSS spectrum, we also tentatively derive upper limits estimates for the unusually weak [OI] $\lambda 6300$ and
Table 2. Parameter values ${ }^{a}$ for $\mathrm{H} \beta$ line diagnostic measures of LAMOST J1131+3114.

\begin{tabular}{lccccc}
\hline \hline Parameter & PM1996 & LAM2011 & APO2012 & Average & $2 \sigma^{b}$ \\
\hline A.I. & 0.079 & 0.085 & 0.086 & 0.083 & 0.160 \\
K.I. & 0.420 & 0.382 & 0.373 & 0.391 & 0.092 \\
$c(1 / 4)$ & 173 & 152 & 24 & 116 & 520 \\
$c(9 / 10)$ & -106 & -128 & -256 & -163 & 320 \\
$c(1 / 4) / F W 0.25 M$ & 0.025 & 0.023 & 0.004 & 0.017 & 0.070 \\
$c(9 / 10) / F W 0.90 M$ & -0.061 & -0.097 & -0.209 & -0.122 & 0.220 \\
\hline
\end{tabular}

Notes. ${ }^{(a)}$ The definition of these parameters is from Marziani et al. (1996). ${ }^{(b)}$ The typical errors is from Zamfir et al. (2010).

[S II] $\lambda \lambda 6717,6731$ fluxes, after removing the $\mathrm{H} \alpha+[\mathrm{N}$ II] background represented by a smooth function, and assuming that the FWHM of these lines was consistent with that of the "base" component. They are given in Table 3 .

Absorption lines of CaII K and $\mathrm{NaI} \mathrm{D}$ were also detected with a redshift consistent with that of the NC (the D doublet is also clearly visible in the spectrum of Eracleous \& Halpern 1994, just shortward of the telluric A bandhead). But Mg I remains undetectable, hence $\mathrm{Ca}$ II and Na I still could be of interstellar origin, arising from dust associated with the NLR region, rather than tracing the host galaxy stellar population.

\section{Discussion and conclusions}

\subsection{The structure of the BLR in LAMOST J1131+3114}

We modeled the $\mathrm{H} \beta$ asymmetric, red extended line profile by the sum of a BC with a $z$ consistent with that of the [O III] base, and a much broader and weaker one, called VBC, largely redshifted with respect to the $\mathrm{BC}$. This $\mathrm{VBC}$ is obvious also in $\mathrm{H} \alpha$ and the tentative fit of the $\mathrm{H} \gamma$ line also confirms its existence. Such a large difference between two subcomponents of the BLR is known in many objects and has been investigated in numerous samples by e.g. Hu et al. (2008) and Zamfir et al. (2010). The latter authors explore the properties of the $\mathrm{H} \beta$ emission line profile in a homogeneous sample of $N \sim 470$ low redshift quasars using composite/median spectra and they measure line profile parameters describing the shape of the broad $\mathrm{H} \beta$ line profile (asymmetry index A.I., centroid shift $c(x)$, and kurtosis index K.I., as defined by Marziani et al. 1996) on the individual objects.

We have measured these parameters on the three best spectra after correcting the $\mathrm{H} \beta$ line from its NLR component and degrading the spectral resolution of the APO spectrum down to the LAMOST spectrum. The results are given in Table 2. Although the APO spectrum $c(i)$ parameters look different, it is difficult to invoke a genuine variation in the observed emission line profile: differences in spectrograph subtended solid angle $\left(1.5^{\prime \prime}\right.$ slit versus $3^{\prime \prime}$ fiber), in sky subtraction, and in flux calibration could lead to inhomogeneous results. Our object is close to the most populated bins of either Population A or Population B in the Zamfir et al. (2010) histograms (their Figs. 7 and 8) without possible clear discrimination based only on these parameters. The total broad $\mathrm{H} \beta$ line FWHM (including VBC) is $4010 \mathrm{~km} \mathrm{~s}^{-1}$. Using the observed flux density at $5100 \AA$ with redshift flux correction and the quasar luminosity distance of $1444 \mathrm{Mpc}$ (with $H_{0}=70 \mathrm{~km} \mathrm{~s}^{-1} \mathrm{Mpc}^{-1}, \Omega_{\mathrm{m}}=0.3$ and $\left.\Lambda=0.70\right)$, we derive a bolometric luminosity of $2.13 \times 10^{46} \mathrm{erg} \mathrm{s}^{-1}$ assuming that $L_{\text {bol }} \sim 10 \lambda L_{5100 \text { A }}$ (Zamfir et al. 2008). The black hole mass $M_{\mathrm{BH}}$ is estimated, after Sulentic et al. (2006), as $5.4 \times 10^{8} M_{\odot}$ and the Eddington luminosity is $7 \times 10^{46} \mathrm{erg} \mathrm{s}^{-1}$, yielding a very high 
Table 3. Emission line ratios and electron temperatures in NLR.

\begin{tabular}{lccc}
\hline \hline Fluxes, line ratios, and $T_{\mathrm{e}}$ & BASE & NC & Global NLR \\
\hline$[\mathrm{O} \mathrm{I}] \lambda 6300^{a}$ & $\leq 55$ & - & - \\
{$[\mathrm{S} \mathrm{II}] \lambda \lambda 6717,6731^{a}$} & $\leq 192$ & - & - \\
{$[\mathrm{O} \mathrm{III}] \lambda 5007 / \mathrm{H} \beta$} & 2.79 & $\geq 14.12$ & 3.52 \\
{$[\mathrm{O}$ III $] \lambda 5007 /[\mathrm{O}$ III $] \lambda 4363$} & 5.90 & $\geq 17.65$ & 7.44 \\
$T_{\mathrm{e} 1}(\mathrm{~K})^{b}$ & 24000 & 12000 & 20000 \\
$T_{\mathrm{e} 2}(\mathrm{~K})^{c}$ & - & 31000 & - \\
{$[\mathrm{O} \mathrm{I}] \lambda 6300 / \mathrm{H} \alpha$} & $\leq 0.055$ & - & $\leq 0.048$ \\
{$[\mathrm{~S} \mathrm{II}] / \mathrm{H} \alpha$} & $\leq 0.18$ & - & $\leq 0.167$ \\
\hline
\end{tabular}

Notes. ${ }^{(a)}$ Flux in $10^{-17} \mathrm{erg} \mathrm{s}^{-1} \mathrm{~cm}^{-2}$. ${ }^{(b)}$ Assuming an electron density $n_{\mathrm{e}}=10^{6} \mathrm{~cm}^{-3}$. ${ }^{(c)}$ Assuming an electron density $n_{\mathrm{e}}=10^{4} \mathrm{~cm}^{-3}$.

Eddington ratio $L_{\mathrm{bol}} / L_{\mathrm{Edd}}$ of $0.3 \pm 0.1$ (with a $L_{5100 \mathrm{~A}}$ assumed uncertainty of $20 \%$ ). We note that alternate derivations (Kaspi et al. 2000, 2005; Wu et al. 2004; Bian et al. 2008) of $M_{\mathrm{BH}}$ based on the total $(\mathrm{BC}+\mathrm{VBC}) \mathrm{H} \beta$ luminosity of $8.9 \times 10^{42} \mathrm{erg} \mathrm{s}^{-1}$ (assuming no internal reddening in the BLR and no flux losses in the spectrographs) lead to $1.4 \times 10^{8} M_{\odot} \leq M_{\mathrm{BH}} \leq 2.6 \times 10^{8} M_{\odot}$ and an even higher Eddington ratio in the range 0.6 to 1.2 .

Instead, these figures place our quasar as a member of Population B defined by Sulentic et al. (2007) and also characterized by Zamfir et al. (2010), this being supported by the radio loudness and weak $\mathrm{Fe}$ II emission. The Eddington ratio, tracing the accretion rate of the central $\mathrm{BH}$, is high for Population $\mathrm{B}$ sources, which could explain the soft X-ray excess (Page et al. 2004), but uncertainty on this parameter is high because of possible continuum boosting and $M_{\mathrm{BH}}$ underestimation.

\subsection{About the NLR}

The question of the physical existence of two separate components, NC and a base in the NLR is open: they are used to formally represent the asymmetry of the [O III] $\lambda \lambda 4959,5007$ lines, but the base is the only significant one in the $\mathrm{H} \beta$ and [O III] $\lambda 4363$ fits. Are the NC and base components physically distinct plasma entities, or is the asymmetry in the [O III] $\lambda \lambda 4959,5007$ lines profile just a consequence of complex internal kinematics of the gas in the NLR? The first hypothesis implies very different excitation and electron temperatures $\left(T_{\mathrm{e}}\right)$ between NC and the base: in Table 3 we report critical line ratios and $T_{\mathrm{e}}$ derived for electron densities of $10^{6} \mathrm{~cm}^{-3}$ (collisional desexcitation limit for the $\lambda 5007$ line) in NC and the base and $n_{\mathrm{e}}=10^{4} \mathrm{~cm}^{-3}$ in NC. We also give these parameters considering a single, global plasma entity for the NLR.

The $\mathrm{H} \beta / \mathrm{H} \gamma$ observed flux ratio is 3.77 , significantly higher than the canonical value of 2.14 , and reflects internal extinction. We thus assume a value of $\sim 4.5$ for $\mathrm{H} \alpha / \mathrm{H} \beta$ and estimate the $\mathrm{H} \alpha$ flux of the base NLR component to $\sim 1000 \times 10^{-17} \mathrm{erg} \mathrm{s}^{-1} \mathrm{~cm}^{-2}$. The derived [O I] $\lambda 6300 / \mathrm{H} \alpha$ and $\left[\mathrm{S}_{\mathrm{II}}\right] / \mathrm{H} \alpha$ line ratios are given in Table 3 as upper limits. Our object is among the weakest in $\left[\mathrm{O}_{\mathrm{I}}\right]$ and $[\mathrm{S} \mathrm{II}]$ in the low- $z$ quasar sample of Eracleous \& Halpern (1994). It appears located at the scarcely populated edge of the region occupied by AGN narrow-line components in standard line ratio diagnostic diagrams, as built by Hao et al. (2005) (Fig. 10), close to the thermal photoionization regime, and thus some contribution from star forming regions to the NLR spectrum cannot be excluded. The [N $\mathrm{NI}_{\mathrm{II}} \lambda 6584$ line is not measurable inside the $\mathrm{H} \alpha$ complex, hence abundance cannot be constrained, as by Huseman et al. (2011), for example.
As usually observed in AGN spectra, the [OIII] $\lambda 4363$ is much brighter than in classical low-density HII regions. Current explanations are based on the combination of normal photoionization with shock excitation, the latter providing specific contributions to some emission line ratios, especially the low ionization ratios. In diagnostic diagrams built from shock models, for example those by Dopita \& Sutherland (1995), or composite shock + photoionization models (see, e.g., Moy \& Rocca-Volmerange 2002) the base component is such that moderate velocity $\left(\sim 100-150 \mathrm{~km} \mathrm{~s}^{-1}\right)$ shock excitation plays a major role, while the $\mathrm{NC}$ is largely dominated by photoionization by the hard UV flux from the central source. If NC and the base do not represent physically separated entities, these line ratios remain similar, and the contribution of moderate velocity shocks remains likely.

Acknowledgements. We are grateful to the anonymous referee for a large number of helpful comments and suggestions which led to improvements in the paper. This study is supported by the National Natural Science Foundation of China under grants No. 10973021, 11233004, 11078019, 61202315. Guoshoujing Telescope (the Large Sky Area Multi-Object Fiber Spectroscopic Telescope LAMOST) is a National Major Scientific Project built by the Chinese Academy of Sciences. Funding for the project has been provided by the National Development and Reform Commission. LAMOST is operated and managed by the National Astronomical Observatories, Chinese Academy of Sciences. The LAMOST Pilot Survey web site is http://data.lamost.org/pdr. M. Eracleous and D. Grupe kindly put their spectrum at our disposal. G.C. expresses his deepest thanks to the Chinese Academy of Sciences for the award of a Visiting Professorship for Senior International Scientists, Grant No. 2010T2J19.

\section{References}

Bian, W. H., Chen, Y. M., Hu, C., Huang, K., \& Xu, Y. 2008, Chin. J. Astron. Astrophys., 8, 522

Brinkmann, W., Siebert, J., Reich, W., et al. 1995, A\&AS, 109, 147

Colla, G., Fanti, C., Ficarra, A., et al. 1970, A\&AS, 1, 281

Corbin, M. R. 1997, ApJS, 113, 245

Cui, X. Q., Zhao, Y. H., Chu, Y. Q., et al. 2012, Res. Astron. Astrophys., 12, 1197

Dopita, M. A., \& Sutherland, R. S. 1995, ApJ, 445, 468

Eracleous, M., \& Halpern, J. P. 1994, ApJS, 90, 1

Eracleous, M., \& Halpern, J. P. 2004, ApJS, 150, 181

Fanti, C., Fanti, R., Ficarra, A., et al. 1975, A\&AS, 19, 143

Giommi, P., Polenta, G., Lähteenmäki, A., et al. 2012, A\&A, 541, A160

Grupe, D., Wills, B. J., Leighly, K. M., et al. 2004, AJ, 127, 156

Grupe, D., Komossa, S., Leighly, K. M., et al. 2010, ApJS, 187, 64

Hao, L., Strauss, M. A., Tremonti, C. A., et al. 2005, AJ, 129, 1783

Helmboldt, J. F., Taylor, G. B., Tremblay, S., et al. 2007, ApJ, 658, 203

Hewitt, A., \& Burbidge, G. 1980, ApJS, 43, 57

Hu, C., Wang, J. M., Ho, L. C., et al. 2008a, ApJ, 683, L115

Husemann, B., Wisotzki, L., Jahnke, K., \& Sanchez, S. F. 2011, A\&A, 535, A72

Iriarte, B., \& Chavira, E. 1957, Bol. Observ. Tonantzintla y Tacubaya, 2, 3

Kaspi, S., Smith, P. S., Netzer, H., et al. 2000, ApJ, 533, 631

Kaspi, S., Maoz, D., Netzer, H., et al. 2005, ApJ, 629, 61

Kellermann, K. I., Sramek, R., Schmidt, M., et al. 1989, AJ, 98, 1195

Kimball, A. E., Ivezić, Ž., Wiita, P. J., et al. 2011, AJ, 141, 182

Luo, A. L., Zhang, Y. X., \& Zhao, Y. H. 2004, Proc. SPIE, 5496, 756

Luo, A. L., Zhang, H. T., Zhao, Y. H., et al. 2012, Res. Astron. Astrophys., 12, 1243

Marziani, P., Sulentic, J. W., Dultzin-Hacyan, D., et al. 1996, ApJS, 104, 37

Marziani, P., Sulentic, J., Zamanov, R., et al. 2003, ApJS, 145, 199

Moy, E., \& Rocca-Volmerange, B. 2002, A\&A, 383, 46

Page, K., Schartel, N., Turner, M., et al. 2004, MNRAS, 352, 523

Shastri, P., Wilkes, B., Elvis, M., et al. 1993, ApJ, 410, 29

Snellen, I., McMahon, R., Hook, I., et al. 2002, MNRAS, 329, 700

Stalin, C., Sagar, R., \& Wiita, P. J. 2004a, J. Astrophys. Astron., 25, 1

Stalin, C., Sagar, R., \& Wiita, P. J. 2004b, MNRAS, 350, 175

Stockman, H. S., Moore, R. L., \& Angel, J. R. P. 1984, ApJ, 279, 485

Stockton, A. 1978, ApJ, 223, 747

Sulentic, J. W., Repetto, P., Stirpe, G. M., et al. 2006, A\&A, 456, 929

Sulentic, J. W., Bachev, R., Marziani, P., et al. 2007, ApJ, 666, 757

Wilkes, B. J., Tananbaum, H., Worrall, D., et al. 1994, ApJS, 92, 53

Wu, X. B., Wang, R., Kong, M. Z., Liu, F. K., \& Han, J. L. 2004, A\&A, 424, 793

Zamfir, S., Sulentic, J. W., \& Marziani, P. 2008, MNRAS, 387, 856

Zamfir, S., Sulentic, J. W., Marziani, P., \& Dultzin, D. 2010, MNRAS, 403, 1759 